\title{
Copy number and targeted mutational analysis reveals novel somatic events in metastatic prostate tumors
}

\author{
Christiane M. Robbins, ${ }^{1}$ Waibov A. Tembe, ${ }^{2}$ Angela Baker, ${ }^{1}$ Shripad Sinari, ${ }^{3}$ \\ Tracy Y. Moses, ${ }^{1}$ Stephen Beckstrom-Sternberg, ${ }^{4}$ James Beckstrom-Sternberg, ${ }^{3}$ \\ Michael Barrett, ${ }^{5}$ James Long, ${ }^{3}$ Arul Chinnaiyan, ${ }^{6}$ James Lowey, ${ }^{2}$ Edward Suh, ${ }^{2}$ \\ John V. Pearson, ${ }^{3}$ David W. Craig, ${ }^{3}$ David B. Agus, ${ }^{7}$ Kenneth J. Pienta, ${ }^{8}$ \\ and John D. Carpten ${ }^{1,9}$ \\ ${ }^{1}$ Division of Integrated Cancer Genomics, Translational Genomics Research Institute, Phoenix, Arizona 85004, USA; ${ }^{2}$ Division \\ of Information Technology, Translational Genomics Research Institute, Phoenix, Arizona, 85004 USA; ${ }^{3}$ Neurogenomics Division, \\ Translational Genomics Research Institute, Phoenix, Arizona 85004, USA; ${ }^{4}$ Pathogen Genomics Division, Translational Genomics \\ Research Institute, Phoenix, Arizona 85004, USA; ${ }^{5}$ Clinical Translational Division, Translational Genomics Research Institute, \\ Scottsdale, Arizona 85259, USA; ${ }^{6}$ Department of Pathology, Howard Hughes Medical Institute, Michigan Center for Translational \\ Pathology, University of Michigan, Ann Arbor, Michigan 48109-0944, USA; ${ }^{7}$ Center for Applied Molecular Medicine, Keck School \\ of Medicine, University of Southern California, Los Angeles, California 90211, USA; ${ }^{8}$ Department of Internal Medicine, University \\ of Michigan Comprehensive Cancer Center, Ann Arbor, Michigan 48109, USA
}

\begin{abstract}
Advanced prostate cancer can progress to systemic metastatic tumors, which are generally androgen insensitive and ultimately lethal. Here, we report a comprehensive genomic survey for somatic events in systemic metastatic prostate tumors using both high-resolution copy number analysis and targeted mutational survey of 3508 exons from 577 cancerrelated genes using next generation sequencing. Focal homozygous deletions were detected at 8p22, 10q23.31, 13q13.1, 13q14.11, and 13q14.12. Key genes mapping within these deleted regions include PTEN, BRCA2, C13ORF15, and SIAH3. Focal high-level amplifications were detected at 5p13.2-p12, 14q21.1, 7q22.1, and Xq12. Key amplified genes mapping within these regions include SKP2, FOXA1, and $A R$. Furthermore, targeted mutational analysis of normal-tumor pairs has identified somatic mutations in genes known to be associated with prostate cancer including $A R$ and TP53, but has also revealed novel somatic point mutations in genes including MTOR, BRCA2, ARHGEF12, and CHD5. Finally, in one patient where multiple independent metastatic tumors were available, we show common and divergent somatic alterations that occur at both the copy number and point mutation level, supporting a model for a common clonal progenitor with metastatic tumor-specific divergence. Our study represents a deep genomic analysis of advanced metastatic prostate tumors and has revealed candidate somatic alterations, possibly contributing to lethal prostate cancer.
\end{abstract}

[Supplemental material is available online at http://www.genome.org. The array CGH data from this study have been submitted to NCl's caArray (https://array.nci.nih.gov/caarray) under experiment ID carpt-00469. The sequencing data from this study have been submitted to the NCBI Sequence Read Archive (http://www.ncbi.nlm.nih.gov/Traces/sra/ sra.cgi) under accession no. SRPO03897 and will be available in $\mathrm{dbGaP.]}$

Prostate cancer still remains the most common male-specific malignancy diagnosed in the United States. In 2009 there were an estimated 192,280 newly diagnosed cases of prostate cancer with 27,360 deaths attributed to this disease (Jemal et al. 2009). Although we have seen slight decreases in incidence rates, primarily due to new methods in estimating new cancer cases by the American Cancer Society, death rates have remained fairly constant between 2007 (27,050) and 2009 (27,360) (Jemal et al. 2007, 2008, 2009). In most cases, these deaths are associated with androgen-insensitive systemic metastatic prostate disease. In light of this incredible clinical problem, there is a growing need to understand the molecular makeup of these lethal tumors. In order to uncover the genomic characteristics of systemic lethal metastatic prostate tumors,

\section{${ }^{9}$ Corresponding author.}

E-mail jcarpten@tgen.org.

Article published online before print. Article and publication date are at http:// www.genome.org/cgi/doi/10.1101/gr.107961.110. we have used high-resolution oligonucleotide array-based comparative genomic hybridization (aCGH) and targeted resequencing using next-generation sequencing technologies in a set of eight tumors from six patients who died of widespread systemic metastatic disease to expose somatic events occurring in lethal cancer.

During the past year, three studies have been reported using genomic profiling to identify somatic copy number alterations specifically in clinical metastatic prostate tumors. In one study, a set of approximately 50 castration-resistant metastatic prostate tumors from 14 patients were analyzed using array aCGH, gene expression profiling, and fluorescence in situ hybridization (FISH) (Holcomb et al. 2009). The results of that study replicated the high frequency of certain alterations such as $8 \mathrm{p}$ loss and $8 \mathrm{q}$ gain, and frequency of chromosome 21 TMPRSS2-ERG fusion deletions in prostate tumors (Holcomb et al. 2009). Their combined analysis of aCGH and gene expression implicated certain genes in metastatic prostate cancer (Holcomb et al. 2009). Finally, they associate specific alterations with metastasis to specific distant sites (Holcomb et al. 
2009). CGH arrays for this study were based on 4200 BAC clones spaced at $>400 \mathrm{~kb}$, whose low resolution decreases the ability to discover focal deletions or amplifications. In a more recent study reported by Liu et al. (2009), high-resolution 1.8 million feature single nucleotide polymorphism (SNP) arrays were used to assess copy number changes in a series of 94 metastatic tumors from 14 patients, where multiple lesions were studied from each patient (Liu et al. 2009). They showed that most, if not all, lesions from each patient shared at least one somatic event, suggesting that metastatic tumors from any given patient were derived from a common progenitor tumor cell (Liu et al. 2009). Furthermore, their data also suggested that there was no obvious relationship between specific lesions and specific systemic metastatic sites (Liu et al. 2009), contrary to results reported by Holcomb et al. (2009). In addition, although high-resolution SNP arrays were used, the only specific alterations reported were TMPRSS2-ERG fusion breakpoint events and $A R$ locus copy number gains. More recently, a very comprehensive genomic study was published for 218 total primary and metastatic tumors, including copy number analysis, gene expression, miRNA analysis, and mutational analysis of exons from more than 100 genes using Sanger sequencing methods (Taylor et al. 2010). In this study, there were five metastatic tumors that were interrogated for both copy number analysis and mutational analysis. The overall results of this study support a significant role for somatic alterations in the gene NCOA2 in prostate cancer (Taylor et al. 2010). Furthermore, they reported an overall low number of somatic point mutations and point to copy number alterations as the most common abnormality affecting prostate tumors. Together these studies have shed significant light on gross copy number changes, have implicated specific regions in metastatic disease, and in a few cases have implicated specific genes that might be involved in metastatic prostate cancer.

A number of candidate genes have been reported that harbor somatic mutations in localized prostate cancer, including $A R$, TP53, KLF6, EPHB2, CHEK2, ZFHX3 (formerly known as ATBF1), and NCOA2 (Newmark et al. 1992; Gottlieb et al. 2004; Huusko et al. 2004; Sun et al. 2005; Dong 2006; Zheng et al. 2006; Agell et al. 2008; Taylor et al. 2010). However, there are limited reports of somatic coding mutations in metastatic tumors. One such example is a study reported by Wong et al. (2007), which describes mutations in the gene encoding plexin- $\mathrm{B}$ in metastatic prostate cancer (Wong et al. 2007); however, as of yet these results have not been replicated in the literature.

Here, we have applied high-resolution aCGH and targeted mutational analysis of a set of known cancer-related genes using next-generation sequencing (NGS) technologies to uncover somatic alterations occurring in the genomes of systemic lethal metastatic prostate tumors. We have replicated copy number alterations known to occur in prostate tumors, including metastatic lesions, and we have also discovered novel focal high-level amplifications and homozygous deletions implicating new genes in metastatic prostate cancer. Furthermore, we have searched extensively for somatic coding mutations in prostate tumors using targeted NGS. Our study has uncovered mutations in known tumor-suppressor genes and oncogenes and has also revealed new candidate genes harboring somatic mutations in lethal metastatic disease.

\section{Results and Discussion}

Our overall sample set is described in Table 1 and consists of eight systemic lethal metastatic tumors and corresponding normal tis-
Table 1. Description of sample set

\begin{tabular}{|c|c|c|c|c|c|}
\hline $\begin{array}{l}\text { Case } \\
\text { no. }\end{array}$ & $\begin{array}{l}\text { Sample } \\
\text { code }\end{array}$ & $\begin{array}{c}\text { Sample } \\
\text { description }\end{array}$ & $\mathrm{CGH}$ & $\begin{array}{l}\text { Targeted } \\
\text { resequencing }\end{array}$ & $\begin{array}{c}\text { Tumor } \\
\text { cellularity }^{a}\end{array}$ \\
\hline RA 37 & RA37T & Dura tumor & Yes & Yes & ND_bone \\
\hline RA37 & RA37N & Dura normal & Yes & Yes & NA \\
\hline RA45 & RA45RM & Rib tumor & Yes & Yes & $80 \%$ tumor \\
\hline RA45 & RA45M & Femur tumor & Yes & Yes & $70 \%$ tumor \\
\hline RA45 & RA45AT & Adrenal tumor & Yes & No & $70 \%$ tumor \\
\hline RA45 & RA45AT2 & Adrenal tumor & No & Yes & $<5 \%$ tumor \\
\hline RA45 & RA45AN & Adrenal normal & Yes & Yes & NA \\
\hline RA48 & RA48RM & Rib tumor & Yes & Yes & ND_bone \\
\hline RA48 & RA48RN & Rib normal & Yes & Yes & ND_bone \\
\hline RA49 & RA49LT & $\begin{array}{l}\text { Lymph node } \\
\text { tumor }\end{array}$ & Yes & Yes & $50 \%$ tumor \\
\hline RA49 & RA49NS & Spleen normal & Yes & Yes & NA \\
\hline RA50 & RA50AT & Adrenal tumor & Yes & No & $75 \%$ tumor \\
\hline RA53 & RA53T & Lung tumor & Yes & Yes & $30 \%$ tumor \\
\hline RA53 & RA53M & Lung normal & Yes & Yes & NA \\
\hline
\end{tabular}

${ }^{a}$ Tumor cellularity was deduced by histological analysis of tissue specimens. ND, Not determined; NA, not applicable.

sue from six unrelated patients with confirmed primary diagnosis of prostate cancer. As tumor cellularity within tissue samples can confound genomic analyses, we quality-controlled our frozen specimens to determine percent tumor content (Table 1).

To search for somatic copy number events in our sample set, we used an array-based comparative genomic hybridization (aCGH) platform built upon roughly 1 million unique genomic oligonucleotide probes, which provides an average probe density of $\sim 3 \mathrm{~kb}$ across the genome. $\log _{2}$ ratios calculated between differentially labeled tumor and reference DNA were plotted for analysis and visualization using Agilent DNA Analytics software. All samples used in the analysis passed quality control as measured by DNA Analytics software (data not shown). Our analyses relied on the ADM-2 algorithm in DNA Analytics software for improved calling of copy number alterations (Lipson et al. 2006). These high-quality and high-resolution data allowed us to focus our copy number analyses on focal somatic copy number events. Focal deletions are defined here as regions of $<5$ million base pairs $(\mathrm{Mb})$ with average $\log _{2}$ ratios for neighboring probes of less than -2 , suggesting homozygous deletion. Focal amplifications are defined here as regions of $<5 \mathrm{Mb}$ with average $\log _{2}$ ratios for neighboring probes of +2 or greater. A list of these regions and detailed descriptions are provided in Table 2.

To discover somatic coding mutations in systemic metastatic prostate tumors, we used Raindance Technologies massive singleplex droplet-based PCR method to amplify a panel of 4002 primer pairs that target 3508 annotated exon sequences from 577 candidate genes in each of our samples. These PCR amplicons were then sequenced using the Applied Biosystems (Life Technologies) SOLiD System NGS technology. The gene set was selected from a panel of genes previously implicated in cancer (Sjoblom et al. 2006) and contains exons from several genes reported to be mutated in prostate cancer, including AR, TP53, EPHB2, CHEK2, NCOA2, and $P L X B 1$. The total amplicon bases (exons and splice junctions) represented $1.63 \mathrm{Mb}$, of which $1.34 \mathrm{Mb}$ represent exon sequences. An analysis pipeline was used that included the BFAST alignment tool (Homer et al. 2009) and variant detection using the tools SolSNP version 1.0 (SA Sinari, A Christoforides, J Beckstrom-Sternberg, A Kurdoglu, JD Carpten, DW Craig, S Beckstrom-Sternberg, JV Pearson, in prep.) and GATK (McKenna et al. 2010) to search for base substitutions and small insertions/deletions (indels). Detailed descriptions of this analysis pipeline are provided in the Supplemental 
Table 2. Regions of focal copy number change in metastatic prostate tumors

\begin{tabular}{|c|c|c|c|}
\hline Focal events & Genomic position $^{a}$ & Genes mapping within region ${ }^{b}$ & Sample \\
\hline $\begin{array}{l}\text { Focal amplification high level } \\
\text { (average } \log _{2} \text { ratio }=+4.0 \text { ) }\end{array}$ & chr5:35716088-36582479 & $\begin{array}{l}\text { SPEF2, IL7R, CAPSL, UGT3A1, UGT3A2, LMBRD2, } \\
\text { MIR580, SKP2, C5orf33, RANBP3L }\end{array}$ & $\begin{array}{l}\text { RA45M RA45RM } \\
\text { RA45AT }\end{array}$ \\
\hline $\begin{array}{l}\text { Focal amplification high level } \\
\text { (average } \log _{2} \text { ratio }=+4.3 \text { ) }\end{array}$ & chr14:36986327-38605977 & MIPOL1, FOXA1, TTC6, SSTR1, CLEC14A, SEC23A & $\begin{array}{l}\text { RA45M RA45RM } \\
\text { RA45AT }\end{array}$ \\
\hline $\begin{array}{l}\text { Focal deletion homozygous } \\
\quad \text { (average } \log _{2} \text { ratio }=-2.3 \text { ) }\end{array}$ & chr13:30557068-33186608 & $\begin{array}{l}\text { HSPH1, B3GALTL, RXFP2, EEF1DP3, FRY, ZAR1L, } \\
\text { BRCA2, N4BP2L1, CG030, N4BP2L2, PDS5B, } \\
\text { KL, STARD13 }\end{array}$ & RA48RM \\
\hline $\begin{array}{l}\text { Focal deletion homozygous } \\
\qquad \text { (average } \log _{2} \text { ratio }=-2.3 \text { ) }\end{array}$ & chr13:40556270-41223477 & $\begin{array}{l}\text { KBTBD6, KBTBD7, MTRF1, NAA16, OR7E37P, } \\
\text { C130RF15, KIAA0564 }\end{array}$ & RA48RM \\
\hline $\begin{array}{l}\text { Focal deletion hemizygous } \\
\quad\left(\text { average } \log _{2} \text { ratio }=-0.6\right)\end{array}$ & chr21:38860275-41778211 & $\begin{array}{l}\text { ERG, NCRNA00114, ETS2, PSMG1, BRWD1, } \\
\text { HMGN1, WRB, LCA5L, SH3BGR, C21 orf88, } \\
\text { B3GALT5, IGSF5, PCP4, DSCAM, C21 orf130, } \\
\text { BACE2, PLAC4, FAM3B, MX2, MX1, TMPRSS2 }\end{array}$ & RA48RM \\
\hline $\begin{array}{l}\text { Focal amplification high level } \\
\text { (average } \log _{2} \text { ratio }=+2.20 \text { ) }\end{array}$ & chr7:102963127-103771360 & RELN, ORC5L & RA49LT \\
\hline $\begin{array}{l}\text { Focal deletion homozygous } \\
\left.\quad \text { (average } \log _{2} \text { ratio }=-3.89\right)\end{array}$ & chr8:15446571-15457837 & $\begin{array}{l}\text { TUSC3 ( } 2 \mathrm{~kb} \text { downstream of exon } 1 \text { within } \\
\text { histone modification region) }\end{array}$ & RA49LT \\
\hline $\begin{array}{l}\text { Focal deletion homozygous } \\
\quad \text { (average } \log _{2} \text { ratio }=-2.56 \text { ) }\end{array}$ & chr10:89566637-90742505 & $\begin{array}{l}\text { CFLP1, KILLIN, PTEN, RNLS, LIPJ, LIPF, LIPK, LIPN, } \\
\text { LIPM, ANKRD22, STAMBPL1, ACTA2 }\end{array}$ & RA49LT \\
\hline $\begin{array}{l}\text { Focal deletion homozygous } \\
\left.\text { (average } \log _{2} \text { ratio }=-2.80\right)\end{array}$ & chr13:45154083-45432647 & SPERT, SIAH3 & RA49LT \\
\hline $\begin{array}{l}\text { Focal amplification high level } \\
\text { (average } \log _{2} \text { ratio }=+2.57 \text { ) }\end{array}$ & chrX:65678672-67093352 & EDA2R, AR & RA49LT \\
\hline $\begin{array}{l}\text { Focal deletion (average } \log _{2} \\
\text { ratio }=-1.14 \text { ) }\end{array}$ & chr21:38801128-41797615 & $\begin{array}{l}\text { ERG, NCRNA00114, ETS2, PSMG1, BRWD1, } \\
\text { HMGN1, WRB, LCA5L, SH3BGR, C21 orf88, } \\
\text { B3GALT5, IGSF5, PCP4, DSCAM, C21 orf130, } \\
\text { BACE2, PLAC4, FAM3B, MX2, MX1, TMPRSS2 }\end{array}$ & RA50T \\
\hline $\begin{array}{l}\text { Focal amplification high level } \\
\quad\left(\log _{2} \text { ratio }=+1.92\right)\end{array}$ & chrX:65549188-66901428 & EDA2R, AR & RA50T \\
\hline
\end{tabular}

${ }^{a}$ Genomic position is based on Human Genome build 36, hg18.

${ }^{\mathrm{b}}$ Gene symbols in bold represent key genes within copy number-altered regions.

Methods. Quality metrics on alignment are provided in Supplemental Table 2. Known SNPs were annotated so that they could be removed from the analysis (data not shown). Annotation was performed on coding variants to identify those that change an amino acid. A final filter was performed to discover those coding variants that were only present in tumor, or were previously shown to be oncogenic. A total of 14 coding mutations were detected, including 12 missense mutations and two nonsense mutations (Table 3). Importantly, all mutations were detected by both SolSNP and GATK. We also detected small $(<5$ bp) indels using GATK; however, after filtering, we did not discover any novel somatic coding indels within our sample set. Visual inspection and further analysis taking into account base quality confirmed that mutations were true positives. Furthermore, several mutations were empirically validated using alternative technologies (Supplemental Fig. 3). To help determine whether nonsynonymous missense mutations were functionally relevant, all mutations were analyzed using SIFT, an algorithm that predicts the likelihood that an amino acid substitution affects protein function based on sequence homology and the physical properties of amino acids (Ng and Henikoff 2001, 2003; Kumar et al. 2009). SIFT analysis results predict that 10 of 12 (83\%) missense mutations detected in our study are damaging and are

Table 3. List of mutations discovered in metastatic prostate tumors

\begin{tabular}{|c|c|c|c|c|c|c|c|c|c|}
\hline $\begin{array}{l}\text { Genomic }^{a} \\
\text { position }\end{array}$ & $\begin{array}{l}\text { Genomic } \\
\text { alleles }^{b}\end{array}$ & Gene ID & $\begin{array}{l}\text { Coding } \\
\text { mutation }\end{array}$ & Amino acid & $\begin{array}{c}\text { Mutation } \\
\text { type }\end{array}$ & $\begin{array}{c}\text { SIFT } \\
\text { prediction }\end{array}$ & Sample & GATK & SolSNP \\
\hline chr1:177345044 & $\mathrm{T} / \mathrm{C}$ & ABL2 & $1936 A>G$ & N646D & Missense & DAMAGING & RA37T & Yes & Yes \\
\hline chr1:6128920 & $\mathrm{G} / \mathrm{T}$ & CHD5 & $1714 C>A$ & R581S & Missense & DAMAGING & RA37T & Yes & Yes \\
\hline chr16:3747299 & $\mathrm{T} / \mathrm{G}$ & CREBBP & $3689 A>C$ & Y1230S & Missense & DAMAGING & RA37T & Yes & Yes \\
\hline chr17:7517878 & $\mathrm{C} / \mathrm{T}$ & TP53 & $785 \mathrm{G}>\mathrm{A}$ & G261D & Missense & DAMAGING & RA45M RA45RM RA45AT2 & Yes & Yes \\
\hline chr1:11104624 & $\mathrm{C} / \mathrm{A}$ & MTOR & $6809 G>T$ & R2270L & Missense & DAMAGING & RA45M RA45RM RA45AT2 & Yes & Yes \\
\hline chrX:66860268 & $\mathrm{C} / \mathrm{T}$ & AR & $2623 C>T$ & $\mathrm{H} 874 \mathrm{Y}$ & Missense & DAMAGING & RA48RM & Yes & Yes \\
\hline chr1:6103883 & $\mathrm{G} / \mathrm{A}$ & CHD5 & $1352 C>T$ & A451V & Missense & TOLERATED & RA49LT & Yes & Yes \\
\hline chr17:7518299 & $\mathrm{T} / \mathrm{C}$ & TP53 & $707 A>G$ & Y236C & Missense & DAMAGING & RA49LT & Yes & Yes \\
\hline chr11:119822381 & $\mathrm{C} / \mathrm{T}$ & ARHGEF12 & $1405 C>T$ & Q469X & Nonsense & NA & RA53T & Yes & Yes \\
\hline chr13:31834694 & $\mathrm{C} / \mathrm{T}$ & BRCA2 & $7840 \mathrm{C}>\mathrm{T}$ & L2614F & Missense & DAMAGING & RA53T & Yes & Yes \\
\hline chr9:129591386 & $\mathrm{G} / \mathrm{A}$ & CDK9 & $862 \mathrm{G}>\mathrm{A}$ & A288T & Missense & DAMAGING & RA53T & Yes & Yes \\
\hline chrX:107863160 & $\mathrm{G} / \mathrm{A}$ & IRS4 & $3071 C>T$ & A1024V & Missense & DAMAGING & RA53T & Yes & Yes \\
\hline chr1:226576309 & $\mathrm{G} / \mathrm{A}$ & OBSCN & $15144 G>A$ & W5048X & Nonsense & NA & RA53T & Yes & Yes \\
\hline
\end{tabular}

${ }^{\mathrm{a}}$ Genomic position is based on Human Genome build 36, hg18.

${ }^{\mathrm{b}}$ First allele represents reference genome allele.

${ }^{c}$ Mutated allele also found in tissue deemed normal (RA48RN). 
likely to confer significant consequences on the encoded proteins (Table 3). However, to understand the significance of these SIFT results, we performed an analysis to determine the rate of "damaging" nonsynonymous changes from a random set of nonsynonymous changes. First, three sets of $\sim 10,000$ random nonsynonymous variants were generated by selecting random lines containing nonsynonymous variants from a list of annotated variant calls from CEU and YRI populations from the 1000 Genomes pilot SNP Calls (030810 Release) (http://www.1000genomes.org). These variants were appropriately formatted for input and submission into SIFT. Upon processing of the result files for damaging variants, we calculated a ratio of the number of damaging variants to the total number submitted to SIFT. Across all datasets, roughly $15 \%$ of the nonsynonymous variants were predicted to be damaging by SIFT. This was in contrast to 10 (damaging) of 12 nonsynonymous mutations (83\%) from our set of mutations detected in metastatic tumor specimens.

All focal somatic copy number changes and key genes affected and mutated are presented in Figure 1. Analysis of our copy number and mutation data further supports a major role for androgen pathway alterations in prostate cancer, including systemic metastatic lesions. Of the six patients studied, we have direct evi- dence of somatic alterations involving the $A R$ gene in three patients, including high-level focal amplification of the $A R$ locus in tumors RA49LT and RA50AT (Table 2; Fig. 1). Although the specific boundaries of these regions are independent in these two tumors, the region was $\sim 1.4 \mathrm{Mb}$ in both tumors and only contained $E D A 2 R$ and $A R$. Furthermore, analysis of mutation data revealed the presence of the known AR H874Y oncogenic mutation in tumor RA48RM (Table 3; Fig. 1). Moreover, tumors from two of the patients with activating events of AR (RA48RM and RA50AT) also contain TMPRSS2-ERG fusion breakpoint events at 21q (Table 2). Interestingly, although R49LT contains high-level amplification at the $A R$ locus, no detectable fusion breakpoint events were detected in this tumor at the genomic level. However, we cannot rule out the presence of fusion transcripts, as they can occur by mechanisms that are not easily detectable at the DNA level (Maher et al. 2009). Furthermore, we detected a clonal focal ( $\sim 1.6 \mathrm{Mb})$ amplicon at $14 \mathrm{q} 21.1$ in three independent metastatic lesions from a single patient (RA45), which encompasses only six known genes (MIPOL1, FOXA1, TTC6, SSTR1, CLEC14A, SEC23A) (Table 2). Foxa transcription factors FOXA1 and FOXA2 interact directly with the androgen receptor and are involved in transcriptional activity of androgen-regulated genes (Gao et al. 2003; Yu et al. 2005).

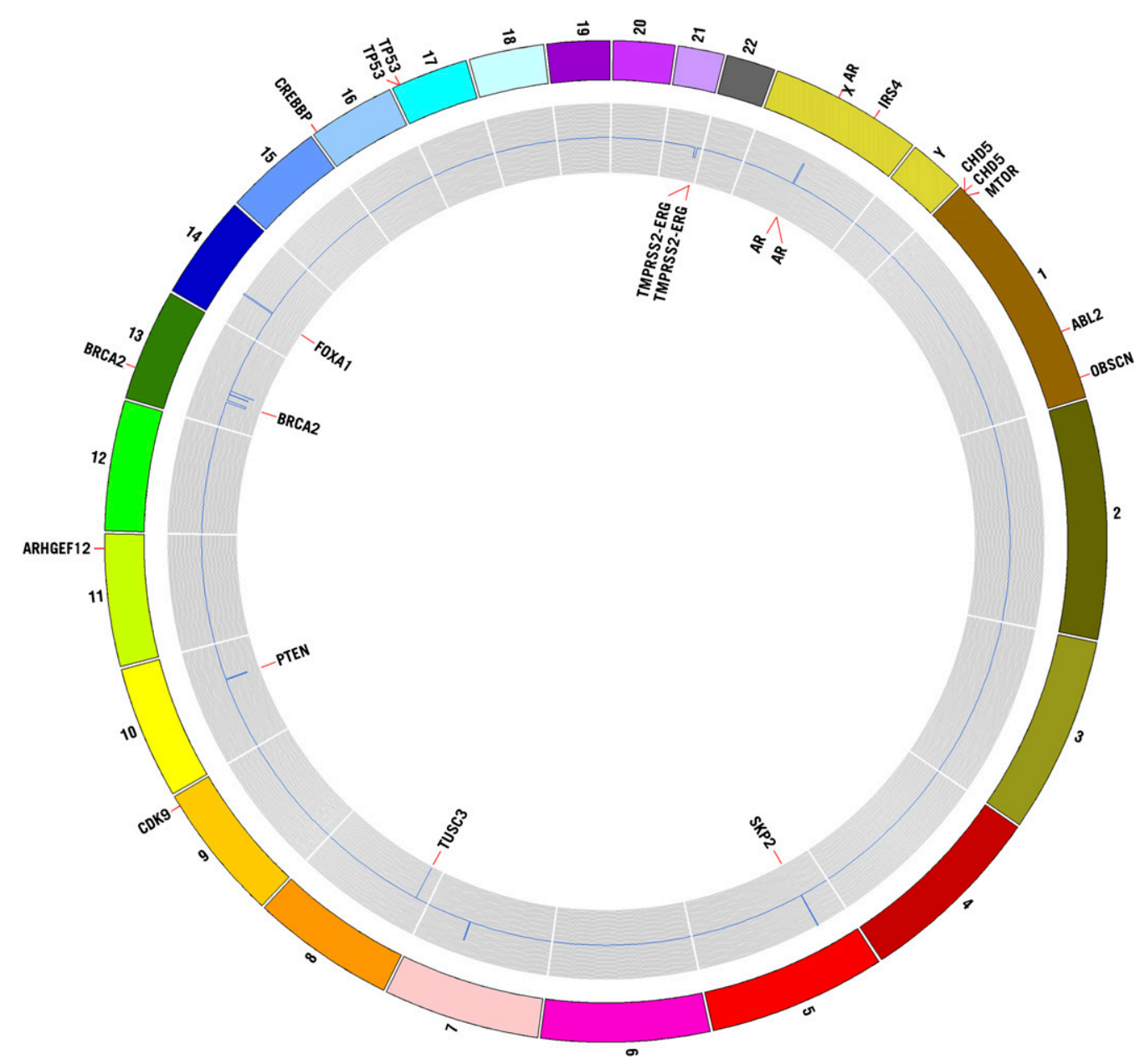

Figure 1. Circos plot illustrating copy number alterations and genes affected by somatic mutation. Regions affected by focal copy number events are plotted within the gray inner circle with deleted regions deviating below copy neutrality (toward center) and amplified regions deviating above copy neutrality. Key genes mapping within copy number alterations are annotated within the innermost portion of the plot. Genes affected by somatic nonsysnonymous mutation are shown at their proper map positions on the outermost area of the Circos plot.

\section{Genome Research} www.genome.org 
Furthermore, increased expression of FOXA1 and FOXA2 has been linked to prostate tumorigenesis and progression (Yu et al. 2005; Mirosevich et al. 2006; van der Heul-Nieuwenhuijsen et al. 2009). Therefore, based on the known functional role of FOXA1 in prostate tumorigenesis, our data would suggest that this gene is a candidate oncogene mapping within the $14 \mathrm{q}$ focal amplicon in tumors from patient RA45. These results provide further evidence for the importance of AR signaling in prostate cancer, which is still of vital importance for the treatment of advanced prostate disease.

We have also discovered a number of other events that contain genes previously associated with important cancer pathways and processes. Several tumors contained alterations affecting the PI3K/AKT pathway. One homozygous deletion in tumor RA49LT encompasses $1.176 \mathrm{Mb}$ at $10 \mathrm{q} 23$ and contains 12 genes, among which is the PTEN tumor-suppressor gene. Furthermore, we have identified a MTOR 6809G $>$ T (R2270L) point mutation in all three metastatic lesions analyzed from patient RA45 (Table 3). MTOR encodes the mammalian target of rapamycin (MTOR), which is a known oncogene and major target of therapeutic drug development for a number of tumor types including androgen-insensitive prostate cancer. A search of the Catalog of Somatic Mutations in the Cancer (COSMIC) database revealed that MTOR mutations have been seen in other tumor types. The R2270L MTOR mutation discovered in our analysis maps within the catalytic site of the MTOR kinase domain, and this amino acid is highly conserved throughout evolution. Moreover, the mutation is predicted as damaging by SIFT analysis. Importantly, this mutation could have significant clinical implications. MTOR inhibitors are under considerable development in phase II clinical trials for treating metastatic prostate cancer, and the presence of the mutation could have positive or negative implications, where the direct activation of MTOR by mutation could render patients either sensitive or resistant to these agents.

Tumors RA45M, RA45RM, and RA45AT, which were derived from the same patient, all exhibit high-level gain at 5p13.2-p12, characterized by a focal $(850 \mathrm{~kb})$ high-level amplicon on the background of a larger region $(8.5 \mathrm{Mb}$ ) of 5 p gain (Fig. 2). The smaller $850-\mathrm{kb}$ region of high-level amplification encompasses only 10 genes, including the SKP2 oncogene. SKP2 is a member of the F-box protein family and interacts with S-phase kinaseassociated protein 1 SKP1 to promote ubiquitination and degradation of proteins, most notably the tumor-suppressor CDKN1B, p27 (Bai et al. 1996; Shibahara 2005). SKP2 has been characterized as an oncogene in a number of tumor types and its overexpression is associated with tumor aggressiveness (Hershko 2008). Specifically, SKP2 overexpression and corresponding down-regulation of p27 has been previously linked to a poor outcome in prostate cancer (Yang et al. 2002; Zheng et al. 2004). Furthermore, a recent link has been established between androgen signaling and SKP2 promoting proliferation of androgen-dependent prostate cancer cells (Wang et al. 2008). In a recent report, 5p gains encompassing SKP2 occur at a significant frequency in prostate cancer (Taylor et al. 2010). Therefore, these data taken together would suggest that copy number gain or high-level amplification of the SKP2 locus, as discovered in our study, is a potential mechanism for up-regulation of SKP2, and would point to SKP2 as a driver of tumorigenesis in tumors from patient RA45.

We have also detected independent somatic alterations of the BRCA2 tumor-suppressor gene, implicating double-strand-break repair defects in metastatic prostate cancer (Fig. 1). One event is defined by a 2.63-Mb homozygous deletion at 13q12.3-q13.2 that encompasses the BRCA2 locus in tumor RA48RM (Table 2). Our analysis has also revealed a BRCA2 7840C $>$ T (L2614F) somatic point mutation in tumor RA53T. This mutation is predicted as damaging by SIFT (Table 3). Of considerable interest is published evidence that up-regulation of SKP2 can result in BRCA2 degradation in prostate tumors (Moro et al. 2006), therefore supporting possible indirect inactivation of BRCA2 in tumors from patient RA45. A recent comprehensive genomic analysis of prostate cancer also revealed somatic alterations of the BRCA2 locus in prostate tumors (Taylor et al. 2010). Taken together, these data suggest that somatic alterations contribute to BRCA2 inactivation in metastatic prostate tumors from three independent patients. These data could have significant clinical and therapeutic implications for metastatic prostate cancer. Recent clinical trials have shown dramatic efficacy for PARP inhibitors in tumors from breast-cancer patients with germline BRCA mutations (Fong et al. 2009). Therefore, deep genomic interrogation of prostate tumors could identify patients with somatic alterations, resulting in BRCA2 inactivation that might be candidates for PARP inhibitors; however, this hypothesis would need to be tested by substantive preclinical studies.

Our study has also revealed additional novel candidate oncogenes and tumor-suppressor genes in lethal metastatic tumors. We identified a focal homozygous deletion at 13q14.12 spanning $279 \mathrm{~Kb}$ in metastatic lesion RA48RM that contained seven genes including C13ORF15 (Table 2), which encodes RGC32. Although RGC32 has been reported as overexpressed in some tumor types (Fosbrink et al. 2005), it has also been strongly implicated as a tumor-suppressor gene in glioma (Saigusa et al. 2007). Saigusa et al. (2007) reported that C13ORF15 mapped within a 13q14.11 region of frequent loss in glioma and showed that RGC32 is induced by p53 in response to DNA damage. They further reported an inverse correlation between C13ORF15 mRNA and tumor grade in primary astrocytomas, especially in tumors with p53 mutation (Saigusa et al. 2007). Furthermore, they demonstrated that overexpression of RGC32 suppressed glioma cell growth in vitro, further supporting its role as a tumor suppressor (Saigusa et al. 2007). An independent 279-kb homozygous deletion at 13q14.12 in another tumor (RA49LT) contained only two genes, including SIAH3, which encodes a member of the seven in absentia (Sina) protein family. These are RING-containing proteins that function as E3 ubiquitin ligases (Hu et al. 1997; Venables et al. 2004). Although SIAH3 has not been characterized functionally, SIAH1 cooperates with SIP (Siah-interacting protein), EBI, and the adaptor protein SKP1, to target beta-catenin for ubiquitination and degradation via a p53-dependent mechanism (Matsuzawa and Reed 2001).

Of the genes somatically mutated in our sample set, only two were mutated in more than one patient, including TP53 and CHD5 (Table 3). CHD5 is a member of the SNF2/RAD54 helicase family, which contains a SWI/SNF-like helicase/ATPase domain, a DNAbinding domain, and a chromodomain involved in chromatin modification for tissue-specific gene expression (Thompson et al. 2003). Importantly, CHD5 has recently been implicated as a tumorsuppressor gene in neuroblastoma, ovarian cancer, and gastric cancer (Bagchi et al. 2007; Gorringe et al. 2008; Wang et al. 2009). Both in vitro and in vivo studies suggest that CHD5 loss is associated with increased proliferation and decreased apoptosis via the p19(ARF)/p53 pathway (Bagchi et al. 2007; Wang et al. 2009). Inactivation of CHD5 can occur through copy number deletion, somatic point mutation, and promoter methylation in other tumor types (Bagchi et al. 2007; Gorringe et al. 2008; Wang et al. 2009). Interestingly, in ovarian cancer, somatic point mutations were reported as heterozygous, where the wild-type allele was inactivated by hypermethylation (Gorringe et al. 2008). This seems 


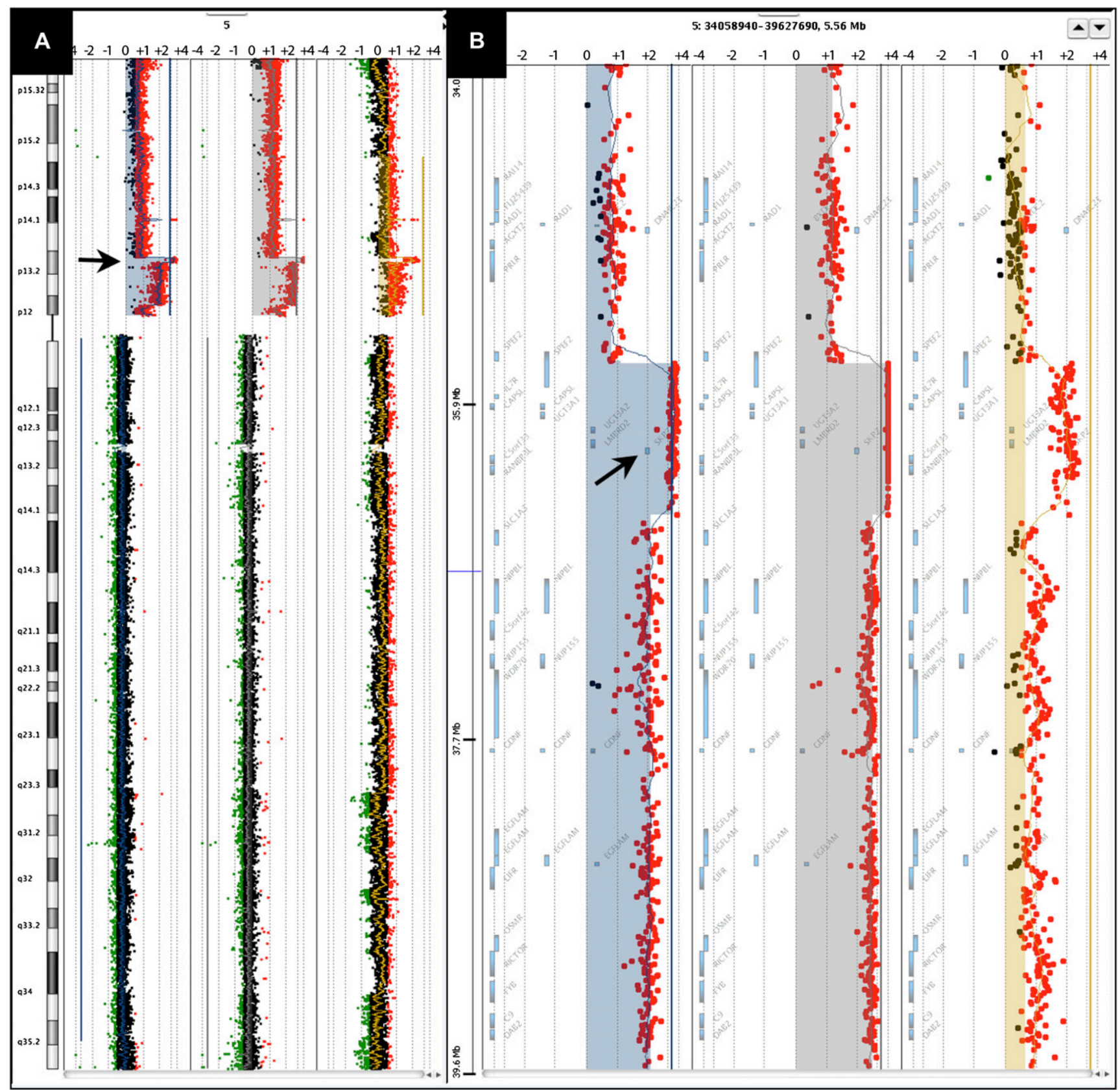

Figure 2. Illustration of high-level amplification at 5p13.2 in tumors from patient RA45. (A) Chromosome view in Agilent DNA Analytics software showing $\log _{2}$ ratios for probes mapping along chromosome 5 for RA45M (left), RA45RM (middle), and RA45AT (right). Amplicon at $5 p 13.2$ is depicted by the arrow. (B) Gene view in DNA Analytics showing $\log _{2}$ ratios for probes mapping to 5p13.2 for RA45M (left), RA45RM (middle), and RA45AT (right). Arrow points to map position of the SKP2 gene locus.

consistent with our findings, as the two mutations discovered in our study were both heterozygous. Our data support a probable role for CHD5 in prostate tumorigenesis. Additionally, a nonsense mutation was discovered in the ARHGEF12 gene, which encodes the leukemia-associated RHO-GEF, LARG. This gene is fused with $M L L$ in acute myeloid leukemia and is believed to play a role in the regulation of RhoA GTPase (Kourlas et al. 2000). Importantly, there is significant evidence in the literature showing that regulation of Rho by LARG is through direct interaction with plexin-B1, which is involved in invasive growth and cell migration, and most impor- tantly, is mutated in metastatic prostate cancer (Aurandt et al. 2002; Hirotani et al. 2002; Swiercz et al. 2002; Wong et al. 2007). Taken together, these data suggest that Rho signaling may be perturbed in metastatic prostate tumors through several mechanisms, among which would be mutations in plexin-B1 or LARG.

We analyzed copy number and mutational analysis data from three independent metastatic lesions from a single patient (RA45) to determine whether or not we could find evidence that these tumors were derived from a common progenitor tumor cell, or whether they were derived from independent tumor cells. 
Systemic sites for these three tumors from patient RA45 can be found in Table 1. Analysis of our genomic data support evidence for a common progenitor clone with site-specific divergence among these three tumors. Evidence supporting the common progenitor models comes from the fact that all three clones share identical high-level focal amplifications at $5 p$ and $14 q$, strongly supporting their derivation from a common ancestral progenitor tumor cell. As stated previously, these are focal events at 5p $(\sim 850$ $\mathrm{kb})$ and $14 \mathrm{q}(\sim 1.6 \mathrm{Mb})$, and harbor only 10 and five genes, respectively. At the copy number state, the $5 \mathrm{p}$ and $14 \mathrm{q}$ amplicons along with 10p whole-arm gain, 10q interstitial deletion, and $8 \mathrm{q}$ whole arm gain represent alterations shared among all three clones. Next generation sequencing data further supports the model for a common progenitor, where mutational analysis revealed common somatic point mutations across all three tumors in both the TP53 and MTOR genes (Table 3). Although data support a common progenitor model for these three metastatic lesions from this patient, further analysis revealed site-specific divergence within the tumors at the three independent sites. Tumors RA45M and RA45RM share several common alterations, including an interstitial deletion at 18q, which is not present in RA45AT (Supplemental Fig. 1). Other common alterations shared only between RA45M and RA45RM include 1q21-q22 gain, 9q34.3 gain, 8p whole arm loss, and a $17 \mathrm{p} 11.2$ interstitial deletion (data not shown). We also uncovered copy number aberrations that are specific to each tumor, including a focal region of gain at 19p12 that is specific to RA45RM, and an interstitial deletion of 21q21 that is specific to RA45M (Supplemental Fig. 1). Taken together, the genomic analysis of these three metastatic lesions from patient RA45 suggest that all three tumors were likely derived from a common progenitor clone. These results would suggest that the oldest clone of the three is RA45AT, as this clone only contains alterations shared among all three clones. Our data also suggest that tumors RA45M and RA45RM probably diverged from a younger clone, as they contain additional common alterations not present in RA45AT, but these two tumors also diverged independently at their respective system sites, evidenced by clonal-specific alterations within RA45M and RA45RM. What we cannot determine from these data is whether the progenitor clone for these three metastatic lesions came from the primary site, or whether the progenitor clone derived at a specific distant site. As access to the primary tumor from this patient is not available, we are unable to reconcile this issue.

In summary, we have completed a detailed genomic analysis of the lethal variety of metastatic prostate tumors. Our study relied on high-resolution aCGH, which uncovered focal copy number events. We also used novel state-of-the-art approaches for targeted mutational analysis facilitated by NGS technologies. These NGS technologies provide a very sensitive and powerful method for detecting mutations even in highly heterogeneous tissue specimens. Our data has validated previous findings in metastatic prostate cancer, including the involvement of specific chromosomal copy number changes and the model of multiple systemic lesions in a single patient being derived from a common progenitor tumor cell. Our study has revealed candidate tumor suppressor genes and oncogenes that are perturbed by copy number change or point mutation that may be implicated in the aggressive lethal phenotype of some prostate tumors. Finally, several of the alterations discovered in our study could have downstream clinical and therapeutic implications for metastatic prostate cancer. Although our study contained a relatively small number of tumors, we have gleaned new insights and taken a glimpse into the genomic landscape of lethal metastatic prostate tumors. However, these data will need to be validated in a larger cohort of patients and tumor specimens in order to search for the true drivers of lethal metastatic disease and to link these alterations to disease progression and understand their role in early disease. Interestingly, a recent study was published describing a comprehensive genomic analysis of more than 200 prostate tumors, including 17 metastatic lesions using CGH and Sanger sequencing methods. Of the 17 metastatic tumors, five were analyzed for copy number changes and mutational analysis (Taylor et al. 2010). Their study implicated a number of genomic regions and genes also discovered in our study such as copy number changes (SKP2, PTEN, TP53) and mutations (BRCA2, TP53). However, our study also has revealed several unique genomic alterations possibly important in lethal metastatic disease. The alterations and candidate genes will need to be functionally validated to confirm their role in prostate tumorigenesis, and their effects on either invasion, migration, or metastatic potential. With the new opportunities for more cost-effective deep whole-genome sequencing, we, and others, are now poised to uncover the full compendium of somatic alterations driving lethal prostate cancer, and perhaps provide new clues on how to best treat these essentially deadly tumors.

\section{Methods}

\section{Clinical specimens and sample processing}

Previously collected (fresh-frozen) metastatic prostate lesions and normal tissue from various anatomical sites were collected through the rapid autopsy program at the University of Michigan (Shah et al. 2004). When applicable, prior to analyte extraction, samples were quality controlled for cellularity (percent tumor) and necrosis.

Soft tissue samples were homogenized, and the sample was treated for $72 \mathrm{~h}$ in proteinase $\mathrm{K}$ at $58^{\circ} \mathrm{C}$ in buffer ATL (Qiagen). DNA extraction was performed with phenol:chloroform using Phase Lock Gel centrifugation tubes (Eppendorf), followed by chromatography using a DNeasy Tissue Kit (Qiagen). We also extracted DNA from tissue specimens using the QiaCube automated sample prep system (Qiagen) following the manufacturer's recommendations. DNA samples were quantified using the Nanodrop 2000 .

\section{Array-based comparative genomic hybridization}

Array CGH was performed on both the Human Genome CGH $244 \mathrm{~K}$ array and the $1 \mathrm{M}$ Human Genome CGH microarray platform for our samples (Agilent Technologies). The procedure used was an adaptation of the protocol recommended by the manufacturer (Keats et al. 2007). Briefly, $1 \mu \mathrm{g}$ of sample genomic DNA and normal female reference (Promega) were digested with Bovine DNase I and directly labeled with either Cy5 or Cy3 using the BioPrime Array CGH Genomic Labeling Module (Invitrogen). Labeled DNA was purified using Vivaspin 500 spin columns (Sartorius). The hybridization reactions containing equal amounts of test and reference DNA were prepared using the Oligo aCGH/ChIP-on-chip Hybridization Kit (Agilent) and were hybridized to the microarray at $65^{\circ} \mathrm{C}$ for $40 \mathrm{~h}$ at a rotation speed of $20 \mathrm{rpm}$. The slides were washed and scanned using the Agilent G2505B DNA microarray scanner. Microarray images were analyzed using Feature Extraction software V10.5.1.1 (Agilent), and $\log _{2}$-transformed ratio data was analyzed with DNA Analytics V4.0.81 (Agilent). Data quality was assessed using the metric values for DLRSpread, signal intensity, and signal-to-noise ratio within Agilent DNA Analytics software. 


\section{Picoliter volume target enrichment}

DNA samples were subjected to whole-genome amplification (WGA) using the GenomiPhi V2 DNA Amplification Kit (GE). Briefly, 10 ng of input DNA was used in each WGA reaction. Amplifications were carried out at $30^{\circ} \mathrm{C}$ for $90 \mathrm{~min}, 65^{\circ} \mathrm{C}$ for $10 \mathrm{~min}$, and held at $4^{\circ} \mathrm{C}$. We performed two independent WGA reactions per sample and pooled the two amplifications to reduce random allele amplification bias. Amplified DNA concentrations were checked using a Nanodrop 2000.

For RainDance library amplification, $10 \mu \mathrm{g}$ of WGA DNA from each sample was sheared by nebulization. The sheared DNA was isopropanol precipitated and further purified using a QIAamp DNA purification kit. The DNA was then quantified by PicoGreen. Subsequently, $3 \mu \mathrm{g}$ of sheared DNA was used in the sequenceenrichment application using the RainDance RDT1000 system. Picoliter volume template droplets containing the sheared DNA were formed on RDT1000 chips and merged with droplets containing a 4000-member primer library that was commercially available from RainDance Technologies. This primer library allows for the PCR amplification of 4005 independent regions, which target 3508 annotated human exons that map to 577 genes. The merged droplets were collected off the chips into a PCR tube for thermocycling. Following PCR amplification, the merged droplet emulsion was broken, followed by a Qiagen MinElute PCR purification column cleanup. The purified amplicon DNA was then analyzed on an Agilent Bioanalyzer.

\section{Next generation sequencing and analysis}

DNA fragment libraries for each of the RainDance-amplified PCR DNA samples were constructed for sequence analysis on the SOLiD version 3.0 next-generation sequencing platform (Applied Biosytems [Life Technologies]) following the manufacturer's instructions. Libraries were constructed using the SOLiD Fragment Library method. Briefly, 200 ng of DNA (RainDance PCR fragments) was randomly fragmented by sonication and subsequently ligated to SOLiD-specific universal sequencing adaptors (P1 and P2). Adaptor-specific primers were used to amplify the library, resulting in roughly $30 \mu \mathrm{g}$ of sequencing library DNA. The amplified library template and proprietary linker-tethered beads were mixed in an emulsion in which each aqueous droplet contains a single bead, a single template strand, and PCR buffer. An emulsion PCR reaction is then carried out, where complementary strands are generated from the bead linker sequences to generate clonal bead populations. After PCR, templates are enriched and modified for deposition onto a glass slide, where massively parallel sequencing occurs by ligation to generate a monoclonal 50-bp sequence read from each templated bead. Based on the size of our target region, sequence coverage, and the number of libraries, an eight-partition deposition chamber for each single flow cell, or octet, was used for each sample. An estimated 30 million P2-positive beads were deposited into each flow cell octet for sequencing.

\section{Next generation sequencing data analysis}

Primary analysis of SOLiD sequencing data consisted of image acquisition in color space. Files containing read and quality data in color space were then used for secondary analysis, including alignment and variant/mutation detection. Alignments were performed against a file containing the target regions using BFAST (Homer et al. 2009). We also performed an alignment against the human genomes (build 36; hg18) using BFAST. For variant detection, we utilized a custom variant calling tool based on a modified Kolmogorov-Smirnov-like statistic (SA Sinari, A Christoforides, J Beckstrom-Sternberg, A Kurdoglu, JD Carpten, DW Craig,
S Beckstrom-Sternberg, JV Pearson, in prep.). The algorithm is nonparametric and makes no assumptions on the nature of the data. It compares the discrete sampled distribution, the pileup on each strand, to the expected distributions (according to ploidy). In the case of a diploid genome, both strands need to provide evidence for the variation. We also used the genome analysis toolkit (GATK) as a secondary pipeline for both single nucleotide variant and indel detection (McKenna et al. 2010). Only nonsynonymous coding mutations detected by both algorithms were characterized for further analysis. To be considered for further analysis, indels would have to be somatic coding indels. Validation of mutations was performed by multiple methods, including the requirement that a mutation be detected by both pipelines, visual inspection of pileups using the Integrated Genomics Viewer, and in some cases, empirically by Sequenom MassARRAY (Supplemental Methods; Supplemental Fig. 2). The program SIFT (Ng and Henikoff 2001, 2003; Kumar et al. 2009) was used to assess possible functional consequences of candidate somatic coding mutations.

\section{Acknowledgments}

This work was supported, in part, by funds from the Prostate Cancer Foundation (J.D.C., D.B.A., K.J.P., A.C.). This work was also supported by the National Institutes of Health Grant CA093900 (K.J.P.), National Institutes of Health SPORE in Prostate Cancer Grant P50 CA69568 (K.J.P, A.C.), Cancer Center Grant P30CA46592 (K.J.P., A.C.), the National Institutes of Health Physical Sciences in Oncology Center Grant U54 CA143907 (D.B.A.), and the American Cancer Society Clinical Research Professorship (K.J.P.). We also thank the High-Performance Biocomputing Center of TGen for providing the clustered computing resources used in this study, which includes the Saguaro-2 cluster supercomputer, a collaborative effort between TGen and the ASU Fulton High Performance Computing Initiative. Saguaro-2 was partially funded by NIH Grant 1S10RR025056-01. We also thank the Care Foundation Desert Mountain, AZ, Mr. and Mrs. K. Earl Durden, and Mr. and Mrs. Eric Tooker for their kind donations to this research (C.M.R., A.B., J.D.C).

\section{References}

Agell L, Hernandez S, de Muga S, Lorente JA, Juanpere N, Esgueva R, Serrano S, Gelabert A, Lloreta J. 2008. KLF6 and TP53 mutations are a rare event in prostate cancer: Distinguishing between Taq polymerase artifacts and true mutations. Mod Pathol 21: 1470-1478.

Aurandt J, Vikis HG, Gutkind JS, Ahn N, Guan KL. 2002. The semaphorin receptor plexin-B1 signals through a direct interaction with the Rhospecific nucleotide exchange factor, LARG. Proc Natl Acad Sci 99: 1208512090.

Bagchi A, Papazoglu C, Wu Y, Capurso D, Brodt M, Francis D, Bredel M, Vogel H, Mills AA. 2007. CHD5 is a tumor suppressor at human 1p36. Cell 128: 459-475.

Bai C, Sen P, Hofmann K, Ma L, Goebl M, Harper JW, Elledge SJ. 1996. SKP1 connects cell cycle regulators to the ubiquitin proteolysis machinery through a novel motif, the F-box. Cell 86: 263-274.

Dong JT. 2006. Prevalent mutations in prostate cancer. J Cell Biochem 97: $433-447$.

Fong PC, Boss DS, Yap TA, Tutt A, Wu P, Mergui-Roelvink M, Mortimer P, Swaisland H, Lau A, O'Connor MJ, et al. 2009. Inhibition of poly(ADPribose) polymerase in tumors from BRCA mutation carriers. NEngl J Med 361: 123-134.

Fosbrink M, Cudrici C, Niculescu F, Badea TC, David S, Shamsuddin A, Shin ML, Rus H. 2005. Overexpression of RGC-32 in colon cancer and other tumors. Exp Mol Pathol 78: 116-122.

Gao N, Zhang J, Rao MA, Case TC, Mirosevich J, Wang Y, Jin R, Gupta A, Rennie PS, Matusik RJ. 2003. The role of hepatocyte nuclear factor-3 alpha (Forkhead Box A1) and androgen receptor in transcriptional regulation of prostatic genes. Mol Endocrinol 17: 1484-1507.

Gorringe KL, Choong DY, Williams LH, Ramakrishna M, Sridhar A, Qiu W, Bearfoot JL, Campbell IG. 2008. Mutation and methylation analysis of the chromodomain-helicase-DNA binding 5 gene in ovarian cancer. Neoplasia 10: 1253-1258. 
Gottlieb B, Beitel LK, Wu JH, Trifiro M. 2004. The androgen receptor gene mutations database (ARDB): 2004 Update. Hum Mutat 23: 527-533.

Hershko DD. 2008. Oncogenic properties and prognostic implications of the ubiquitin ligase Skp2 in cancer. Cancer 112: 1415-1424.

Hirotani M, Ohoka Y, Yamamoto T, Nirasawa H, Furuyama T, Kogo M, Matsuya T, Inagaki S. 2002. Interaction of plexin-B1 with PDZ domaincontaining Rho guanine nucleotide exchange factors. Biochem Biophys Res Commun 297: 32-37.

Holcomb IN, Young JM, Coleman IM, Salari K, Grove DI, Hsu L, True LD, Roudier MP, Morrissey CM, Higano CS, et al. 2009. Comparative analyses of chromosome alterations in soft-tissue metastases within and across patients with castration-resistant prostate cancer. Cancer Res 69: 7793-7802.

Homer N, Merriman B, Nelson SF. 2009. BFAST: An alignment tool for large scale genome resequencing. PLOS ONE 4: e7767. doi: 10.1371/journal. pone.0007767

Hu G, Chung YL, Glover T, Valentine V, Look AT, Fearon ER. 1997. Characterization of human homologs of the Drosophila seven in absentia (sina) gene. Genomics 46: 103-111.

Huusko P, Ponciano-Jackson D, Wolf M, Kiefer JA, Azorsa DO, Tuzmen S, Weaver D, Robbins C, Moses T, Allinen M, et al. 2004. Nonsensemediated decay microarray analysis identifies mutations of EPHB2 in human prostate cancer. Nat Genet 36: 979-983.

Jemal A, Siegel R, Ward E, Murray T, Xu J, Thun MJ. 2007. Cancer statistics, 2007. CA Cancer J Clin 57: 43-66.

Jemal A, Siegel R, Ward E, Hao Y, Xu J, Murray T, Thun MJ. 2008. Cancer statistics, 2008. CA Cancer J Clin 58: 71-96.

Jemal A, Siegel R, Ward E, Hao Y, Xu J, Thun MJ. 2009. Cancer statistics, 2009. CA Cancer J Clin 59: 225-249.

Keats JJ, Fonseca R, Chesi M, Schop R, Baker A, Chng WJ, Van Wier S, Tiedemann R, Shi CX, Sebag M, et al. 2007. Promiscuous mutations activate the noncanonical NF-kappaB pathway in multiple myeloma. Cancer Cell 12: 131-144.

Kourlas PJ, Strout MP, Becknell B, Veronese ML, Croce CM, Theil KS, Krahe R, Ruutu T, Knuutila S, Bloomfield CD, et al. 2000. Identification of a gene at 11q23 encoding a guanine nucleotide exchange factor: Evidence for its fusion with MLL in acute myeloid leukemia. Proc Natl Acad Sci 97: 2145-2150.

Kumar P, Henikoff S, Ng PC. 2009. Predicting the effects of coding nonsynonymous variants on protein function using the SIFT algorithm. Nat Protoc 4: 1073-1081.

Lipson D, Aumann Y, Ben-Dor A, Linial N, Yakhini Z. 2006. Efficient calculation of interval scores for DNA copy number data analysis. J Comput Biol 13: 215-228.

Liu W, Laitinen S, Khan S, Vihinen M, Kowalski J, Yu G, Chen L, Ewing CM, Eisenberger MA, Carducci MA, et al. 2009. Copy number analysis indicates monoclonal origin of lethal metastatic prostate cancer. Nat Med 15: 559-565.

Maher CA, Kumar-Sinha C, Cao X, Kalyana-Sundaram S, Han B, Jing X, Sam L, Barrette T, Palanisamy N, Chinnaiyan AM. 2009. Transcriptome sequencing to detect gene fusions in cancer. Nature 458: 97-101.

Matsuzawa SI, Reed JC. 2001. Siah-1, SIP, and Ebi collaborate in a novel pathway for beta-catenin degradation linked to p53 responses. Mol Cell 7: 915-926.

McKenna A, Hanna M, Banks E, Sivachenko A, Cibulskis K, Kernytsky A, Garimella K, Altshuler D, Gabriel S, Daly M, et al. 2010. The Genome Analysis Toolkit: A MapReduce framework for analyzing nextgeneration DNA sequencing data. Genome Res 20: 1297-1303.

Mirosevich J, Gao N, Gupta A, Shappell SB, Jove R, Matusik RJ. 2006. Expression and role of Foxa proteins in prostate cancer. Prostate 66: 1013-1028.

Moro L, Arbini AA, Marra E, Greco M. 2006. Up-regulation of Skp2 after prostate cancer cell adhesion to basement membranes results in BRCA2 degradation and cell proliferation. J Biol Chem 281: 22100-22107.

Newmark JR, Hardy DO, Tonb DC, Carter BS, Epstein JI, Isaacs WB, Brown TR, Barrack ER. 1992. Androgen receptor gene mutations in human prostate cancer. Proc Natl Acad Sci 89: 6319-6323.
Ng PC, Henikoff S. 2001. Predicting deleterious amino acid substitutions. Genome Res 11: 863-874.

Ng PC, Henikoff S. 2003. SIFT: Predicting amino acid changes that affect protein function. Nucleic Acids Res 31: 3812-3814.

Saigusa K, Imoto I, Tanikawa C, Aoyagi M, Ohno K, Nakamura Y, Inazawa J. 2007. RGC32, a novel p53-inducible gene, is located on centrosomes during mitosis and results in G2/M arrest. Oncogene 26: 1110-1121.

Shah RB, Mehra R, Chinnaiyan AM, Shen R, Ghosh D, Zhou M, Macvicar GR, Varambally S, Harwood J, Bismar TA, et al. 2004. Androgenindependent prostate cancer is a heterogeneous group of diseases: Lessons from a rapid autopsy program. Cancer Res 64: 9209-9216.

Shibahara T, Onishi T, Franco OE, Arima K, Sugimura Y. 2005. Downregulation of Skp2 is correlated with p27-associated cell cycle arrest induced by phenylacetate in human prostate cancer cells. Anticancer Res 25: $1881-1888$.

Sjoblom T, Jones S, Wood LD, Parsons DW, Lin J, Barber TD, Mandelker D, Leary RJ, Ptak J, Silliman N, et al. 2006. The consensus coding sequences of human breast and colorectal cancers. Science 314: 268-274.

Sun X, Frierson HF, Chen C, Li C, Ran Q, Otto KB, Cantarel BL, Vessella RL, Gao AC, Petros J, et al. 2005. Frequent somatic mutations of the transcription factor ATBF1 in human prostate cancer. Nat Genet 37: 407-412.

Swiercz JM, Kuner R, Behrens J, Offermanns S. 2002. Plexin-B1 directly interacts with PDZ-RhoGEF/LARG to regulate RhoA and growth cone morphology. Neuron 35: 51-63.

Taylor BS, Schultz N, Hieronymus H, Gopalan A, Xiao Y, Carver BS, Arora VK, Kaushik P, Cerami E, Reva B, et al. 2010. Integrative genomic profiling of human prostate cancer. Cancer Cell 18: 11-22.

Thompson PM, Gotoh T, Kok M, White PS, Brodeur GM. 2003. CHD5, a new member of the chromodomain gene family, is preferentially expressed in the nervous system. Oncogene 22: 1002-1011.

van der Heul-Nieuwenhuijsen L, Dits NF, Jenster G. 2009. Gene expression of forkhead transcription factors in the normal and diseased human prostate. BJU Int 103: $1574-1580$.

Venables JP, Dalgliesh C, Paronetto MP, Skitt L, Thornton JK, Saunders PT, Sette C, Jones KT, Elliott DJ. 2004. SIAH1 targets the alternative splicing factor T-STAR for degradation by the proteasome. Hum Mol Genet 13: 1525-1534.

Wang H, Sun D, Ji P, Mohler J, Zhu L. 2008. An AR-Skp2 pathway for proliferation of androgen-dependent prostate-cancer cells. J Cell Sci 121: 2578-2587.

Wang X, Lau KK, So LK, Lam YW. 2009. CHD5 is down-regulated through promoter hypermethylation in gastric cancer. J Biomed Sci 16: 95. doi: 10.1186/1423-0127-16-95.

Wong OG, Nitkunan T, Oinuma I, Zhou C, Blanc V, Brown RS, Bott SR, Nariculam J, Box G, Munson P, et al. 2007. Plexin-B1 mutations in prostate cancer. Proc Natl Acad Sci 104: 19040-19045.

Yang G, Ayala G, De Marzo A, Tian W, Frolov A, Wheeler TM, Thompson TC, Harper JW. 2002. Elevated Skp2 protein expression in human prostate cancer: Association with loss of the cyclin-dependent kinase inhibitor p27 and PTEN and with reduced recurrence-free survival. Clin Cancer Res 8: $3419-3426$

Yu X, Gupta A, Wang Y, Suzuki K, Mirosevich J, Orgebin-Crist MC, Matusik RJ. 2005. Foxa1 and Foxa2 interact with the androgen receptor to regulate prostate and epididymal genes differentially. Ann N Y Acad Sci 1061: 77-93.

Zheng XY, Ding W, Xie LP, Chen ZD. 2004. [Correlation of Skp2 and P27kip1 protein expression and clinicopathological features of prostate cancer]. Chinese J Cancer 23: 215-218.

Zheng L, Wang F, Qian C, Neumann RM, Cheville JC, Tindall DJ, Liu W. 2006. Unique substitution of CHEK2 and TP53 mutations implicated in primary prostate tumors and cancer cell lines. Hum Mutat 27: 10621063.

Received March 22, 2010; accepted in revised form October 6, 2010. 


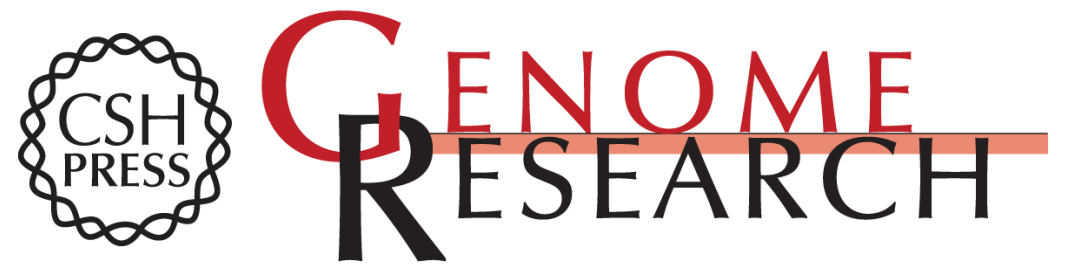

\section{Copy number and targeted mutational analysis reveals novel somatic events in metastatic prostate tumors}

Christiane M. Robbins, Waibov A. Tembe, Angela Baker, et al.

Genome Res. 2011 21: 47-55 originally published online December 8, 2010

Access the most recent version at doi:10.1101/gr.107961.110

Supplemental Material

References

License

Email Alerting Service
http://genome.cshlp.org/content/suppl/2010/11/09/gr.107961.110.DC1

This article cites 49 articles, 13 of which can be accessed free at: http://genome.cshlp.org/content/21/1/47.full.html\#ref-list-1

Receive free email alerts when new articles cite this article - sign up in the box at the top right corner of the article or click here.

\section{Affordable, Accurate Sequencing.}

